\title{
Emergence of Biaxial Nematic Phases in Solutions of Semiflexible Dimers
}

\author{
Arvin Vaghela ${ }^{1}$, Paulo I. C. Teixeira ${ }^{2,3}$, and Eugene M. Terentjev ${ }^{1}$ \\ ${ }^{1}$ Cavendish Laboratory, University of Cambridge, \\ J. J. Thomson Avenue, Cambridge CB3 OHE, United Kingdom \\ ${ }^{2}$ ISEL - Instituto Superior de Engenharia de Lisboa, Instituto Politécnico de Lisboa \\ Rua Conselheiro Emídio Navarro 1, 1959-007 Lisbon, Portugal and \\ ${ }^{3}$ Centro de Física Teórica e Computacional \\ Faculdade de Ciências da Universidade de Lisboa \\ Campo Grande, Edifício C8, 1749-016 Lisbon, Portugal
}

\begin{abstract}
We investigate the isotropic, uniaxial nematic and biaxial nematic phases, and the transitions between them, for a model lyotropic mixture of flexible molecules consisting of two rigid rods connected by a spacer with variable bending stiffness. We apply density-functional theory within the Onsager approximation to describe strictly excluded-volume interactions in this athermal model and to selfconsistently find the orientational order parameters dictated by its complex symmetry, as functions of the density. Earlier work on lyotropic ordering of rigid bent-rod molecules is reproduced, and extended to show explicitly the continuous phase transition at the Landau point, at a critical bend angle of $36^{\circ}$. For flexible dimers with no intrinsic biaxiality, we nevertheless find that a biaxial nematic phase can form at sufficiently high density and low bending rigidity. For bending stiffness $\kappa>0.86 k_{B} T$, this biaxial phase manifests itself as dimer bending fluctuations occurring preferentially in one plane. When the dimers are more flexible, $\kappa<0.86 k_{B} T$, the modal shape of the fluctuating dimer is a ' $\mathrm{V}$ ' with a sharp opening angle, and the biaxial order parameter changes sign. These two regions are separated by a narrow strip of uniaxial nematic in the phase diagram, which we generate in terms of the variables spacer stiffness and particle number density.
\end{abstract}

PACS numbers: $64.70 . \mathrm{Md}, 61.30 . \mathrm{Cz}$

\section{INTRODUCTION}

The discovery in 1996 that achiral molecules of bentrod shape could exhibit ferroelectric smectic behavior [1] started a decades-long fascination with these molecules, which are now known variably as bent-core, 'bananas', boomerangs, bow-shaped or V-shaped [2]. Their positionally disordered liquid crystal (LC) phases were thoroughly and systematically catalogued by Lubensky and Radzihovsky [3]. Interestingly, these include the elusive biaxial nematic $\left(\mathrm{N}_{\mathrm{B}}\right)$ phase, which is characterized by preferential alignment of not one, but two mutually orthogonal molecular axes, and thus exhibits two directors. First predicted by Freisier back in 1970 [4], the $\mathrm{N}_{\mathrm{B}}$ phase has since been a source of excitement in LC science, on account of both its fundamental interest and its potential applications. For example, bistable displays or devices might exploit the easier orientability of the secondary director of the $\mathrm{N}_{\mathrm{B}}$ phase to achieve faster switching times. The theory, simulation and experiment of biaxial nematics have been extensively and thoroughly reviewed in [5], whereas the molecular design of biaxial nematogens is addressed in [6].

In order to realize biaxial nematic order, the molecules need to have a corresponding symmetry, with two distinct orthogonal axes of anisometry. The parallelepiped has been the most common starting point $[4,7]$, yet bent rods were also early candidates $[8,9]$. Biaxiality was indeed claimed experimentally in 2004 by Madsen et al. [10] and Acharya et al. [11], as well as more recently by others [12-14], though not without some controversy [15-17].
Two theoretical approaches have been developed to investigate the phase behavior of bent-rod molecules, which are suitable for thermotropic and lyotropic mesogens respectively. In the first of these $[18,19]$, particles are assumed to interact via a continuous anisotropic potential of the appropriate symmetry, which is then treated within a mean-field approximation. The second approach [8], in contrast, models each particle as two hard spherocylinders joined at their ends at a (fixed) angle, including excluded-volume interactions only; the system is then treated at the level of the Onsager second-virial approximation. Despite their different starting points, both theories make essentially the same predictions. This is not unexpected if one recognises that, from the point of view of orientational order, the density in a lyotropic $\mathrm{LC}$ plays the same role as the inverse temperature in a thermotropic LC. We shall return to this point later, but in summary: at high temperatures (low densities), the stable phase is isotropic (I); as the temperature is lowered (or the density raised), straight or slightly bent rods with inter-arm angles close to $180^{\circ}$ will undergo a first-order transition into a calamitic uniaxial nematic phase $\left(\mathrm{N}_{\mathrm{U}}^{+}\right)$, where their long axes are ordered. Conversely, for strongly bent rods with an inter-arm angle close to $90^{\circ}$, the first-order transition is into a discotic (oblate) uniaxial nematic phase $\left(\mathrm{N}_{\mathrm{U}}^{-}\right)$, where it is now the axes perpendicular to the plane of the two arms that order. In between these limits, for an inter-arm angle close to the tetrahedral angle, there is a Landau point at which the isotropic phase moves directly into a biaxial nematic phase $\left(\mathrm{N}_{\mathrm{B}}\right)$ via a continuous transition. Continuous transitions from the $\mathrm{N}_{\mathrm{U}}^{+}$and $\mathrm{N}_{\mathrm{U}}^{-}$phases into the 
$\mathrm{N}_{\mathrm{B}}$ phase are also predicted on either side of the Landau point.

Experimentally, however, the $\mathrm{N}_{\mathrm{B}}$ phase was seen to occur at higher temperatures and smaller rod bending angles (hence smaller molecular biaxialities) than predicted. One possible source of this discrepancy might be the large permanent transverse electric dipole present in bent dimers $[10,20,21]$, which was not accounted for in $[8,18,19]$. There have been several attempts to incorporate dipoles into the theory $[22,23]$, which have yielded results generally pointing in the right direction, as well as new and exciting phase diagram topologies including polar biaxial phases. Still, in the present paper we are mostly concerned with qualitative effects rather than quantitative agreement, and so we shall ignore these complications.

Early Monte Carlo simulations of bent-rod particles composed of two hard spherocylinders did not find unambiguous evidence for a $\mathrm{N}_{\mathrm{B}}$ phase, because of the formation of pairs of particles locked together [24], or found it to be pre-empted by smectic phases for aspect ratio $L / D=5[25]$. Similar results were obtained for molecules consisting of seven tangent Lennard-Jones spheres rigidly arranged into a V-shape [26]. However, Monte Carlo simulations of an extension of the Lebwohl-Lasher lattice model of nematogens did confirm the general topology of the phase diagram, with a Landau point at the tetrahedral inter-arm angle for symmetrical bent rods [27]; in asymmetrical bent rods, the Landau point was shifted to smaller angles and eventually disappeared for large asymmetries.

In this paper we revisit the simple model of a molecular dimer with a flexible joint connecting the two rigid rods, see Fig. 1. The instantaneous shape of a dimer is a bent rod, but we assume its equilibrium shape to be straight, with an energy penalty associated with spacer bending. Flexibility of the molecular bond allows for a more general description of semiflexible filaments and other molecules with an internal degree of freedom. The meanfield density-functional theory of flexible thermotropic dimers was developed by Terentjev et al. [28, 29], who focused on the properties of the elastic spacer connecting the two equivalent rods, and looked at even-odd effects and the values of elastic constants [30-32]. However, the analysis in $[28,29]$ was restricted to the uniaxial nematic phase. Luckhurst [33] formulated a mean-field theory of flexible thermotropic dimers that includes all symmetries and all types of orientational order. Yet this approach leads to problems in treating the excluded volume when the single-particle probabilities for each rod are correlated; moreover, it has not been used to make any detailed calculations of transitions. Our purpose is to address all these issues for the model of flexible dimers, where the control parameter is the bending stiffness of the joint (spacer). In the limit of infinite stiffness our theory must reduce to that of the standard lyotropic nematic phase of rigid rods of doubled length. We do not consider the more complicated problem of flexible dimers (a)

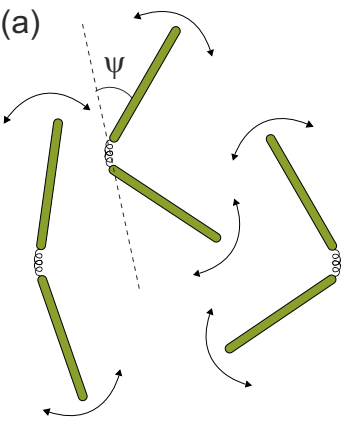

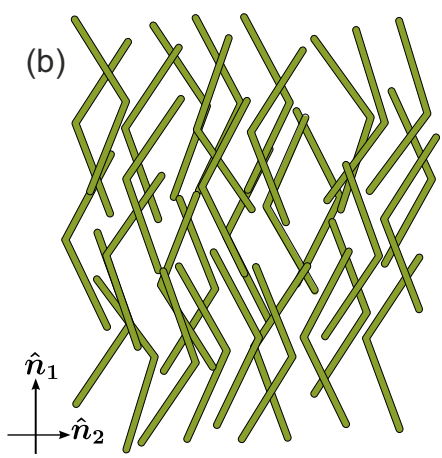

FIG. 1. (a) Flexible dimers consisting of two hard rods connected by a flexible spacer. The angle $\psi$, with equilibrium value $\psi=0$, measures the degree of bending. (b) The biaxial nematic phase of rigid bent rods studied in [8].

that are bent at a finite angle $\psi_{0}$ in equilibrium (see [29] for a basic treatment of this problem in the uniaxial-order limit).

This paper is organised as follows: in section II we recapitulate the theory of [8] for rigid bent rods (II A) and generalise it to include a flexible joint (IIB). In section III we present our numerical results for the key order parameters, and the associated phase diagrams, for both rigid bent rods and flexible dimers. The existence of a biaxial phase in a solution of flexible but intrinsically uniaxial dimers, with a non-trivial topology of phase diagram, is the main result of this paper.We conclude in section IV.

\section{THEORY}

\section{A. Rigid bent rods}

In this section we recapitulate the theory of [8]. In the Onsager second-virial theory, the free energy density of a nematic phase can be written as [34, 35]:

$$
\frac{F(T, V, \rho)}{N k_{B} T}=\ln \left(\Lambda^{3} \rho\right)-1+\int f(\Omega) \ln f(\Omega) d \Omega+B_{2} \rho,
$$

where $\rho=N / V$ is the (number) density of rods. $\Lambda=$ $\left(2 \pi \hbar^{2} / m k_{B} T\right)^{1 / 2}$ is the thermal de Broglie wavelength, $\Omega=(\phi, \theta, \chi)$ the set of Euler angles [36] defining the passive rotation that transforms the particle-fixed frame $\{u, v, w\}$ into the lab-fixed frame $\{x, y, z\}$, and $f(\Omega)$ is the single-particle orientational distribution function (ODF). The first two terms in the right-hand side of Eq. (1) are the ideal-gas contribution. The third term is due to the loss of entropy caused by the orientational order: it vanishes in the isotropic phase. Finally, the last term is the contribution due to interparticle interactions, where $B_{2}$ is the (angle-dependent) second virial coefficient:

$$
B_{2}=-\frac{1}{2 V} \int \Phi_{12} f\left(\Omega_{1}\right) f\left(\Omega_{2}\right) d \Omega_{1} d \Omega_{2} d \mathbf{r}_{1} d \mathbf{r}_{2} .
$$


In Eq. $2, V$ is the volume of the system and $\Phi_{12}$ is the Mayer $f$-function

$$
\Phi_{12}=e^{-\phi_{12} / k_{B} T}-1,
$$

in which $\phi_{12}=\phi\left(\mathbf{r}_{1}, \mathbf{r}_{2}, \Omega_{1}, \Omega_{2}\right)$ is the interparticle pair potential, a function of both the positional $\left(\mathbf{r}_{i}\right)$ and orientational $\left(\Omega_{i}\right)$ coordinates of the two interacting particles.

It should be noted that the 2nd-virial approximation only takes into account interactions between no more than two particles simultaneously, and is therefore only reliable at low densities. Discussions of the validity of Onsager theory are usually framed in terms of how large the particle aspect ratio $L / D$ must be such that higherorder virial coefficients are negligible compared to $B_{2}$. As shown by Allen et al. [37], this is only the case beyond $L / D \sim 10^{2}$. However, Bolhuis and Frenkel [38] found from simulations that Onsager theory actually gives a very good description of the isotropic-nematic (I-N) transition densities of hard spherocylinders for $L / D>20$. Recalling that the I-N transition in the Onsager limit occurs at a reduced density $c \approx 3.5$ (see the definition in Eq. (22) below), this suggests that Onsager theory should in practice be valid for volume fractions below $\varphi \approx 3.5 / 20=0.175$ or number densities below $\rho D^{3} \approx 0.01$. We stress that this is merely an operational criterion and not a rigorous proof of validity, which is still an open question.

In the case of 'hard' particles with no long-range interaction potential, the Mayer $f$-function $\Phi_{12}=-1$ for overlapping geometries (when $\phi_{12}=\infty$ ), and $\Phi_{12}=0$ otherwise $\left(\phi_{12}=0\right)$. Hence, $B_{2}$ reduces to the ODF-weighted integral over orientations of the (angle-dependent) excluded volume $v_{\text {excl }}(1,2)$ of two particles:

$$
B_{2}=\frac{1}{2} \int f\left(\Omega_{1}\right) v_{\mathrm{excl}}\left(\Omega_{1}, \Omega_{2}\right) f\left(\Omega_{2}\right) d \Omega_{1} d \Omega_{2} .
$$

This latter function is not known analytically for any non-convex shapes, and thus needs to be found numerically. Bisi and Rosso [39] calculated the excluded volume of two V-shaped objects consisting of tangent hard spheres by Monte Carlo integration, and derived an approximation to it in terms of symmetry-adapted Wigner functions. We could use this result, but a simpler alternative yielding qualitatively correct behavior is to follow [8] and approximate the excluded volume of two dimers by the sum of excluded volumes of pairs of their constituent hard rods, here taken to be hard spherocylinders of length $L$ and diameter $D$. This 'superposition approximation' amounts to treating the spherocylinders as independent particles, and will introduce errors of (leading) order $L D^{2}$. These will, however, be vanishingly small relative to the main 'unperturbed' term (of order $D L^{2}$ [40]) in the limit of long, thin spherocylinders, $L / D \gg 1$, where Onsager theory is best applicable. Onsager theory itself predicts a $\mathrm{I}-\mathrm{N}$ transition at a density $\sim 1 / D L^{2}$ (if $L / D$ is of order 5 or smaller, it becomes necessary to include higher-order virial coefficients [41]). Finally, we

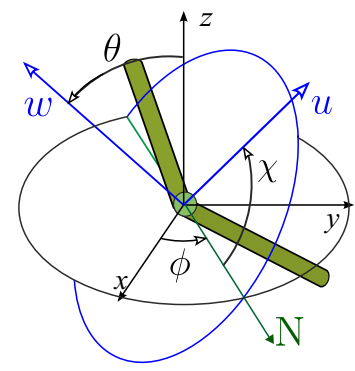

FIG. 2. The principal axes and standard Euler angles defining the orientation of a biaxial bent-rod molecule in the lab-fixed frame $(x, y, z)$. The shape of the molecule can be embedded in a parallelepiped of thickness $D$, with long principal axis $\boldsymbol{w}$ and short principal axis $\boldsymbol{u} \perp \boldsymbol{w}$. The long axis is inclined at the angle $\theta$ from $z$, the short axis lies in the plane that crosses the $x-y$ plane at angle $\phi$, and $\boldsymbol{u}$ is rotated counter-clockwise by angle $\chi$ in this plane.

TABLE I. Table of excluded volumes of two bent rods in the Onsager limit $(L \gg D)$, where the first rod is aligned with the laboratory frame and the particle-fixed axes of the second are coincident with the laboratory axes. Refer to Fig. 2 for the definition of Euler angles $(\theta, \phi, \chi)$. The first column shows in which plane of the $(x, y, z)$ frame the pair of orthogonal principal vectors $\boldsymbol{w}, \boldsymbol{u}$ lies, for each case.

\begin{tabular}{|lll|lll|l|}
\hline$x$ & $y$ & $z$ & $\phi$ & $\theta$ & $\chi$ & $v_{\text {excl }} / 2 D L^{2}$ \\
\hline$u$ & - & $w$ & 0 & 0 & 0 & $2|\sin 2 \psi|$ \\
$w$ & - & $u$ & 0 & $90^{\circ}$ & 0 & $2(1+|\cos 2 \psi|)$ \\
- & $w$ & $u$ & $90^{\circ}$ & $90^{\circ}$ & 0 & $4\left|\sin \left(\cos ^{-1}\left(\frac{1}{2} \sin 2 \psi\right)\right)\right|$ \\
- & $u$ & $w$ & $90^{\circ}$ & 0 & 0 & $4\left|\sin \left(\cos ^{-1}\left(\cos ^{2} \psi\right)\right)\right|$ \\
$u$ & $w$ & - & $90^{\circ}$ & $90^{\circ}$ & $90^{\circ}$ & $4\left|\sin \left(\cos ^{-1}\left(\sin ^{2} \psi\right)\right)\right|$ \\
$w$ & $u$ & - & 0 & $90^{\circ}$ & $90^{\circ}$ & $4\left|\sin \left(\cos ^{-1}\left(\frac{1}{2} \sin 2 \psi\right)\right)\right|$ \\
\hline
\end{tabular}

do not recover the correct limit when the inter-arm angle goes to zero (angle $\psi=\pi / 2$ in Fig. 1), a point we revisit in section III A.

Instead of working with the angle-dependent excluded volume in its general form, we shall follow Straley [7] by considering the values this excluded volume takes for a few particular orientations of the two particles. Assuming particle 1 to be located at the origin of the lab frame, i.e. $\left(\theta_{1}, \phi_{1}, \chi_{1}\right)=(0,0,0)$ (see Fig. 2), we compute the excluded volumes for the six distinct orientations particle 2 can have subject to the constraint that its long and short axes are directed along the coordinate axes. Note that within our superposition approximation we are neglecting shape polarity, which is addressed in [42], for example. These orientations are listed in Table I, where we have used the result that the excluded volume of two long, thin $(L / D \gg 1)$ spherocylinders is [34]

$$
v_{\mathrm{excl}}(\theta) \simeq 2 D L^{2} \sin (\pi-2 \psi)
$$

which depends upon the angle between their long axes, cf. Fig. 1(a). In the spirit of Straley's treatment [7], we then fit the entries in Table I to a second-order 'interpolating potential', which can be writen as an expansion in terms of symmetry-adapted basis functions. Following 
the notation of Mulder [35],

$$
V_{\text {int }}(\Omega)=\alpha+\beta \Delta_{1}(\theta)+\gamma\left[\Delta_{2}(\phi, \theta)+\Delta_{3}(\theta, \chi)\right]+\delta \Delta_{4}(\phi, \theta, \chi),
$$

where the angular functions $\Delta_{i}(\Omega)$ are linear combinations of Wigner rotation functions of rank-2 [35, 36], given by

$$
\begin{aligned}
& \Delta_{1}(\theta)=P_{2}(\cos \theta)=\frac{1}{2}\left(3 \cos ^{2} \theta-1\right), \\
& \Delta_{2}(\phi, \theta)=\sin ^{2} \theta \cos 2 \phi, \\
& \Delta_{3}(\theta, \chi)=\sin ^{2} \theta \cos 2 \chi, \\
& \Delta_{4}(\phi, \theta, \chi)=\frac{1}{2}\left(1+\cos ^{2} \theta\right) \cos 2 \phi \cos 2 \chi \\
& -\cos \theta \sin 2 \phi \sin 2 \chi .
\end{aligned}
$$

The coefficients $(\alpha, \beta, \gamma, \delta)$ in Eq. (6) are chosen such that $V_{\text {int }}(\Omega)$ is equal to the excluded volume when the principal axes of the molecules are coincident with the coordinate frame (as given in Table I):

$$
\begin{aligned}
\frac{\alpha}{2 D L^{2}}= & \frac{2}{3}\left(|\sin 2 \psi|+4\left|\sin \left(\cos ^{-1}\left(\frac{1}{2} \sin 2 \psi\right)\right)\right|\right), \\
\frac{\beta}{2 D L^{2}}= & \frac{4}{3}\left(|\sin 2 \psi|+\left|\sin \left(\cos ^{-1}\left(\frac{1}{2} \sin 2 \psi\right)\right)\right|\right)-1 \\
& -|\cos 2 \psi|-2\left|\sin \left(\cos ^{-1}\left(\sin ^{2} \psi\right)\right)\right| \\
\frac{\gamma}{2 D L^{2}}= & \frac{1}{2}\left(1+|\cos 2 \psi|-2\left|\sin \left(\cos ^{-1}\left(\sin ^{2} \psi\right)\right)\right|\right) \\
\frac{\delta}{2 D L^{2}}= & 1+|\cos 2 \psi|+2\left|\sin \left(\cos ^{-1}\left(\sin ^{2} \psi\right)\right)\right| \\
& -4\left|\sin \left(\cos ^{-1}\left(\frac{1}{2} \sin 2 \psi\right)\right)\right|
\end{aligned}
$$

We stress that these expressions are only valid in the Onsager limit, i.e. thin rods for which $L \gg D$. This interpolation approximation preserves the qualitative behavior of the true excluded-volume potential whilst greatly simplifying the form of the interaction and the subsequent algebra.

The basis functions (7) also define the four orientational order parameters:

$$
\begin{aligned}
& S_{1}=\left\langle\Delta_{1}(\theta)\right\rangle, \quad S_{2}=\left\langle\Delta_{2}(\phi, \theta)\right\rangle, \quad S_{3}=\left\langle\Delta_{3}(\theta, \chi)\right\rangle, \\
& S_{4}=\left\langle\Delta_{4}(\phi, \theta, \chi)\right\rangle,
\end{aligned}
$$

where the angular brackets denote an ODF-weighted angular average. It is important to note that, over the years, the four key order parameters have been defined in many different ways. A comprehensive historical and comparative analysis has been undertaken by Rosso [43]. Here we adopt the notation of the first correct analysis, by Straley [7], though giving the four order parameters different (perhaps more systematic) names.

The 'usual' uniaxial nematic order is, of course, described by $S_{1}$. Non-zero $S_{1}$ and $S_{3}$, combined with zero $S_{2}$ and $S_{4}$, indicate a uniaxial nematic phase aligned along the z-axis. In contrast, nonzero $S_{2}$ and $S_{4}$ describe biaxiality emerging in the plane perpendicular to the principal $z$-axis. Positive $S_{1}, S_{3}$ indicate a 'prolate' $\mathrm{N}_{\mathrm{U}}^{+}$phase (the typical ordering of rods), whilst negative $S_{1}, S_{3}$ correspond to an 'oblate' $\mathrm{N}_{\mathrm{U}}^{-}$phase (the ordering of flat discs). It should be noted that multiple nonzero $S_{i}$ may be indicative of a uniaxial phase that forms about some axis other than $z$ (rather than a biaxial phase). This stems from our earlier arbitrary choice of $z$-axis. Results must therefore be interpreted carefully, as we shall see in section III. In general the system will pass through a uniaxial phase before exhibiting any biaxiality except at the Landau point, which by definition is the point in the phase diagram where the isotropic phase transitions directly to $\mathrm{N}_{\mathrm{B}}$. A more thorough discussion of this can be found in [19]. Finally, it should be noted that the set of four order parameters in Eq. (9) is appropriate for a biaxial nematic phase without polarity [42]. More sophisticated theories exist that predict many other phases of polar bent dimers; in particular, Lubensky and Radzihovsky [3], Méttout [44], and Luckhurst et al. [45] have pointed out that the usual description of order in terms of a single second-rank tensor is insufficient to capture the full richness of phases potentially found in these systems. In this paper we restrict ourselves to non-polar nematic phases.

Note that $V_{\text {int }}(\Omega)$ in Eq. (6) is in fact a two-particle potential; as in $[7,8]$ we have assumed that the reference particle is located at the origin of the lab-fixed frame (both in terms of position and alignment). The corresponding single-particle mean-field potential, $W(\Omega)$, is then obtained by integration over the coordinates of one of the particles: $W_{M F}\left(\Omega_{1}\right)=\int V_{\text {int }}\left(\Omega_{1}, \Omega_{2}\right) f\left(\Omega_{2}\right) d \Omega_{2}$. By symmetry, its form is determined by the same basis functions

$$
W_{M F}=w_{1} \Delta_{1}+w_{2} \Delta_{2}+w_{3} \Delta_{3}+w_{4} \Delta_{4},
$$

leading to the self-consistency relations

$$
\begin{aligned}
& w_{1}=\beta S_{1}+\gamma S_{3}, \quad w_{2}=\frac{3}{4} \beta S_{2}+\gamma S_{4}, \\
& w_{3}=\gamma S_{1}+\frac{3}{4} \delta S_{3}, w_{4}=\gamma S_{2}+\delta S_{4} .
\end{aligned}
$$

From Eq. (1), the mean-field approximation to the free energy is thus

$$
\begin{aligned}
\frac{F}{N k_{B} T}= & \ln \left(\Lambda^{3} \rho\right)-1+\int f(\Omega) \ln (f(\Omega)) d \Omega \\
& +\frac{\rho}{2}\left(w_{1} S_{1}+w_{2} S_{2}+w_{3} S_{3}+w_{4} S_{4}\right) .
\end{aligned}
$$

Minimisation of this free energy functional with respect to the ODF $f(\Omega)$ yields

$$
f(\Omega)=\frac{1}{Z} \exp \left(-\frac{1}{2} \rho W_{M F}(\Omega)\right),
$$

with the normalisation constant

$$
Z=\int \exp \left(-\frac{1}{2} \rho W_{M F}(\Omega)\right) d \Omega .
$$


Substituting Eq. (13) into Eq. (1) and recalling that $f_{\text {iso }}(\Omega)=1 / 8 \pi^{2}$ in the isotropic phase, we find the excess free energy (that is, the difference in free energy between the nematic and isotropic phases)

$$
\frac{\Delta F}{N k_{B} T} \equiv \frac{F_{\text {nem }}-F_{\text {iso }}}{N k_{B} T}=\ln \left(\frac{8 \pi^{2}}{Z}\right) .
$$

We see that the equilibrium free energy difference is completely encapsulated in the normalisation constant $Z$ (the partition function of the nematic mean field).

We finish this section with a remark on the formal similarity of the Onsager and Maier-Saupe theories of lyotropic and thermotropic nematics, respectively: both lead to a self-consistency equation of the form of Eq. (13). There is a critical difference however, in that the factor of density $(\rho)$ multiplying the mean field in the Onsager theory is replaced by $1 / k_{B} T$ in the Maier-Saupe theory. This reflects the fact that the Maier-Saupe theory deals with attractive forces in addition to configurational entropies and so allows for a temperature-driven phase transition, in contrast to the density-driven Onsager transition caused by entropic excluded-volume effects. In fact, both the Onsager and Maier-Saupe theories can be viewed as special cases of more general mean-field theories, where both the density $\rho$ and the Boltzmann factor $1 / k_{B} T$ act as control parameters. The limits $\rho \rightarrow 0$ $\left(k_{B} T \rightarrow \infty\right)$ and $\rho \rightarrow \infty\left(k_{B} T \rightarrow 0\right)$ correspond to the (non-interacting) ideal gas and strong-coupling behavior, respectively. Consequently, all results reported in this paper for athermal systems can be transposed onto a thermally-driven system by making the substitution $\rho \rightarrow 1 / k_{B} T$, and so have wider validity than may at first be evident.

\section{B. Flexible dimers}

Here we extend the above treatment to the case of a flexible, jointed dimer as shown in Fig. 1(a). The dimer is modelled as two rods connected by a springlike bond, with an energy penalty for nonzero bond angle

$$
U_{\text {bend }}=\frac{1}{2} \kappa \psi^{2} \text {. }
$$

This can be incorporated into the mean-field formalism developed above. First, on adding a new degree of freedom to the system we must include its (configurational) entropic contribution. We do this by extending the ODF and measure

$$
f(\Omega) d \Omega \rightarrow f(\Omega, \psi) d \Omega d \psi,
$$

such that the new entropy is contained within the Gibbs term in Eq. (1). Next, the bending energy, Eq. (16), contributes an additional term to the overall free energy

$$
\Delta F_{\psi}=N \frac{1}{2} \kappa\left\langle\psi^{2}\right\rangle
$$

We now define an additional pair of parameters

$$
w_{5}=\frac{\kappa}{\rho k_{B} T}, \quad \Delta_{5}=\psi^{2},
$$

and update the molecular mean-field expression, which can be written concisely as

$$
W_{M F}=w_{1} \Delta_{1}+w_{2} \Delta_{2}+w_{3} \Delta_{3}+w_{4} \Delta_{4}+w_{5} \Delta_{5} .
$$

The total free energy now changes from Eq. (12) to the form

$$
\begin{aligned}
\frac{F}{N k_{B} T}= & \ln \left(\Lambda^{3} \rho\right)-1 \\
& +\int f(\Omega, \psi) \ln (f(\Omega, \psi)) d \Omega d \psi \\
& +\frac{\rho}{2}\left(w_{1} S_{1}+w_{2} S_{2}+w_{3} S_{3}+w_{4} S_{4}+w_{5} S_{5}\right) .
\end{aligned}
$$

Note that $S_{5}=\left\langle\Delta_{5}\right\rangle$ does not represent a new order parameter, being used merely as a common convenient notation (a measure of the mean-square fluctuation of the bending angle). Similarly, the added bending contribution $\left(w_{5} S_{5}\right)$ is not a virial term as the four other terms are, but an intra-molecular energy. This common notation allows streamlined integration into the generic Straley method. We set the true isotropic ODF $f_{\text {iso }}(\Omega, \psi)=1 / 8 \pi^{3}$, in keeping with Eq. (13); the extra factor of $\pi$ arising from the integration over $\psi$.

Finally, there is a subtlety in Eq. (20) in that all factors $\left\{w_{i}\right\}$ depend on the bending angle $\psi$ through the coefficients $\{\beta, \gamma, \delta\}$, cf. Eq. (8), and so must also be averaged with the ODF $f(\Omega, \psi)$ when solving the self-consistent field condition Eq. (13).

\section{RESULTS AND DISCUSSION}

It will be helpful for what follows to define a dimensionless 'reduced density' as

$$
c \equiv \frac{\pi}{4} D L^{2} \rho=\frac{L}{D} \frac{N}{V} \frac{\pi}{4} D^{2} L=\frac{L}{D} \varphi,
$$

where $\varphi$ is the volume fraction of the rods. This follows earlier works $[8,40,46]$ and uses the fact that the proper volume of a (straight) rod is $\frac{\pi}{4} D^{2} L$ to leading order in $D / L \ll 1$. Onsager theory can be used to find equilibria between phases of different densities (see, e.g. [40]). We shall not do this here: instead, we investigate the stability of different phases as a function of density.

\section{A. Rigid dimers}

The self-consistent mean-field condition, Eq. (13), was solved at each reduced density $c$ by making an initial guess for the set of order parameters $\left\{S_{i}\right\}$, substituting 

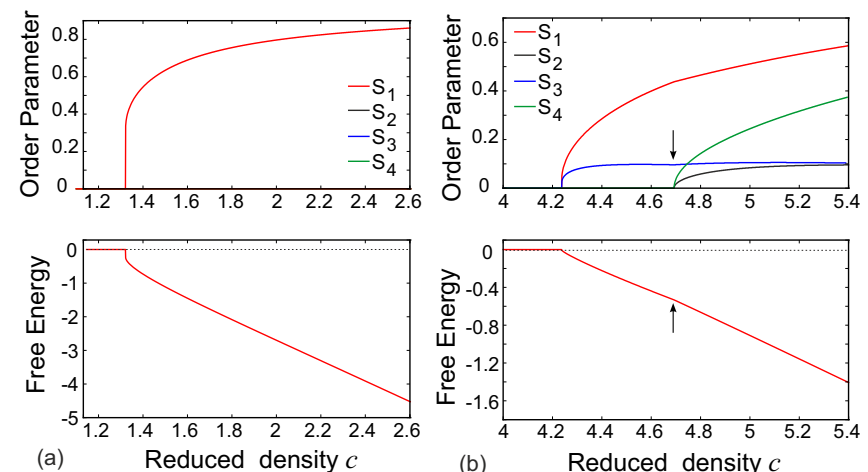

FIG. 3. (a) Order parameter and excess free energy (in units of $k_{B} T$ ) plotted for $\psi=0^{\circ}$. There is a strong first-order phase transition, with only $S_{1}$ present. (b) Order parameter and excess free energy (in units of $k_{B} T$ ) for $\psi=36^{\circ}$ reflect phase transitions just below the Landau point. The $\mathrm{I}-\mathrm{N}_{\mathrm{U}}^{+}$ transition is very nearly continuous, reflecting the proximity to the Landau point. The arrows highlight the singularities observed at the $\mathrm{N}_{\mathrm{U}}-\mathrm{N}_{\mathrm{B}}^{+}$transition.

them into them into the mean field $W_{M F}(10)$, and recalculating $\left\{S_{i}\right\}$ through Eqs. (9). These values were resubstituted back into Eq. (13), and the process iterated until the convergence condition

$$
\left|S_{i}-S_{i}^{\text {old }}\right|<10^{-7}
$$

was satisfied for all order parameters. This was deemed sufficiently stringent to ensure full convergence. The numerical integration was performed using 16-point GaussLegendre quadrature, implemented in Fortran [47]. Tabulated vales of trigonometric functions were used when performing the angular integrations in order to increase efficiency.

We have reproduced the results of Teixeira et al. [8], correcting minor algebraic errors in their analysis in the process. Figure 3(a) shows the first-order phase transition found for a straight double-rod $(\psi=0)$. The functional form of $W_{M F}$ (in particular that of the $\Delta_{1}$ part, corresponding to the primary uniaxial order parameter) indicates that this is identical to the Maier-Saupe result if one were to replace $\rho \rightarrow 1 / k_{B} T$. As the bond angle increases, the phase transition occurs at progressively higher densities; in addition, the secondary uniaxial order parameter $S_{3}$ becomes finite, see Fig. 3(b). A softening of the phase transition is also evident, becoming 'weakly first-order' in nature [49] as the rigid bond angle increases. This is related to the increasing biaxiality of the molecules [19, 48], illustrated by the rise in $S_{2,4}$. In fact, the transition is predicted to become continuous at the Landau point - as indeed is observed in Fig. 5 below.

A qualitative change in behavior is observed at the Landau point which, for this system, is at $\psi_{\text {Landau }} \sim$ $36.3^{\circ}$. For $\psi<\psi_{\text {Landau }}$, the system exhibits a phase transition from isotropic to a prolate $\mathrm{N}_{\mathrm{U}}^{+}$phase, whereas for $\psi>\psi_{\text {Landau }}$ the transition is into an oblate uniaxial phase $\mathrm{N}_{\mathrm{U}}^{-}$phase. The relevant results for $\psi$ just below
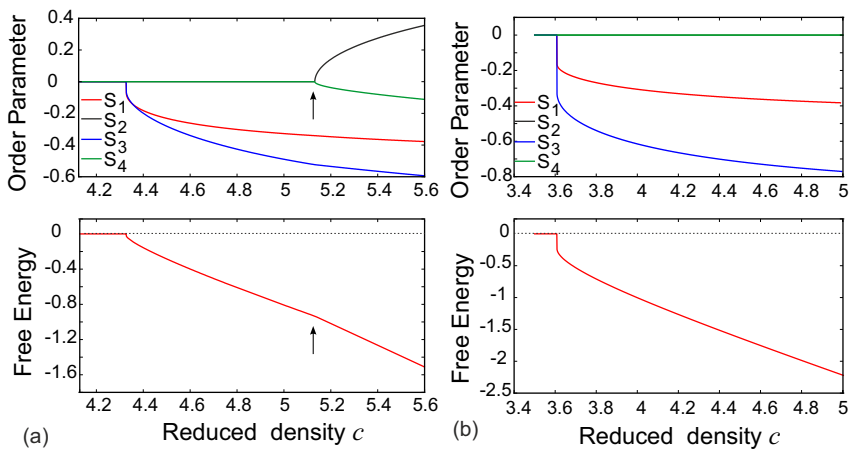

FIG. 4. (a) Order parameter and excess free energy (in units of $k_{B} T$ ) reflect phase transitions for $\psi=37^{\circ}$, just above the Landau point. The first transition here is to an oblate uniaxial $\mathrm{N}_{\mathrm{U}}^{-}$phase; the arrows highlight the singularities at the $\mathrm{N}_{\mathrm{U}^{-}}$ $\mathrm{N}_{\mathrm{B}}$ transition. (b) For a large bending angle, $\psi=45^{\circ}$, the molecule is essentially oblate and the corresponding $\mathrm{N}_{\mathrm{U}}^{-}$phase is the only outcome in the range of densities investigated.
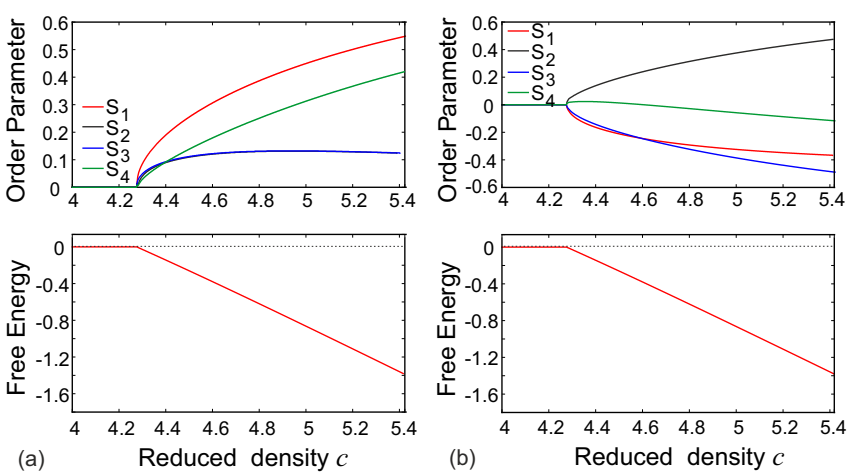

FIG. 5. I-N $\mathrm{N}_{\mathrm{B}}$ phase transitions for $\psi=36.32^{\circ}$, at the Landau point. Plot (a) was obtained with all initial $\left\{S_{i}\right\}$ positive; plot (b) with negative initial $S_{1}$ and $S_{2}$. The transition is manifestly continuous, directly from the isotropic to the biaxial nematic phase, and the free energy of both resulting phases is nearly the same (to $\sim 10^{-3} k_{B} T$ per molecule). This is consistent with the change in behavior from prolate- to oblate-like order across the Landau point, but implies that there are two degenerate biaxial phases at the point itself, $\mathrm{N}_{\mathrm{B}}^{+}$and $\mathrm{N}_{\mathrm{B}}^{-}$.

and just above the Landau point are given in Figs. 3(b) and 4(a), respectively. The main difference is in the sign of $S_{1}$ (i.e., prolate vs. oblate uniaxial order), which then guides the difference in the corresponding biaxial phase $\mathrm{N}_{\mathrm{B}}^{+}$and $\mathrm{N}_{\mathrm{B}}^{-}$, respectively. When solving Eq. (13) one may find local minima, depending on the initial guesses for the parameters $\left\{S_{i}\right\}$. This is the case for $\psi>37^{\circ}$, where positive guesses for the order parameters lead to a phase for which $S_{2}=2 S_{1}, S_{4}=\frac{2}{3} S_{3}$, indicating $\mathrm{N}_{\mathrm{U}}^{+}$ order about the $x$-axis. However, this is a metastable phase, higher in free energy than the $\mathrm{N}_{\mathrm{U}}^{-}$phase observed when one chooses negative initial $S_{1}, S_{3}$ and positive $S_{2}$, $S_{4}$. This is clearly illustrated in Fig. 4 (b) for $\psi=45^{\circ}$. The equilibrium free energy difference $\Delta F$ (per particle), given by the Eq. (15), is included for completeness, and to illustrate the nature of the transitions. 
It is important to note that the interpolating-potential approximation, Eq. (6) and coefficients, Eq. (8), does not recover the correct limit as $\psi \rightarrow \pi / 2$ - a fully-folded dimer. This might be regarded (to leading order in $D / L$ ) as a straight parallelepiped of length $L$ and thicknesses $2 D$ in one plane and $D$ in the other. This is inherent in the nature of the approximation, which becomes increasingly inaccurate as the bond angle reaches this limit [8] because of the asymmetry associated with the (arbitrary) choice of $z$-axis. As previously discussed, however, this model does give the correct limit as $\psi \rightarrow 0$ (the straight double-rod). Despite this shortcoming, the approximation preserves the topology of the phase diagram and the character of the transitions whilst providing a tractable theory, and is therefore very useful.

\section{B. Flexible dimers}

In this case we have the dimer bending angle $\psi$ as an additional fluctuating variable, with associated changes in the energy and entropy. As one would expect, by increasing the dimension of the phase space over which each integration takes place, and because of the complexity of the coefficients Eqs. (8) (which now need to be integrated over $\psi$ ), computation of the solution becomes significantly more demanding. Fortunately the basis functions $\Delta_{1-4}$ are functions of $\Omega$ only, and the coefficients $\{\beta, \gamma, \delta\}$ are functions of $\psi$ only, allowing the relevant contributions to $w_{1-4}$ to be calculated separately for efficiency. Tabulated trigonometric values were again used when performing the integration over Euler angles $\Omega$. However, the coefficients given by Eqs. (8) were evaluated using double-precision trigonometric functions, since the solution of Eq. (13) was found to be very sensitive to the accuracy of their computation, occasionally not converging when tabulated values were employed. In addition, the Gauss-Legendre quadrature used to perform the integrations over solid angle was found to be insufficiently accurate as $\kappa \rightarrow \infty$, being unable to capture the rapid change of the integrand with $\psi$. Consequently, the Romberg method [47] was employed for integration over the internal bond angle.

Furthermore, the first-order nature of the transition allowed the convergence condition, Eq. (23), to be relaxed somewhat when producing these data. Taking $S_{i}>0.1$ as the criterion for a phase transition, the condition $\left|S_{i}-S_{i}^{\text {old }}\right|<10^{-5}$ was sufficient to produce smooth phase boundaries. Small sections of data were checked with the more stringent condition, Eq. (23), in order to verify the validity of this relaxation.

Figure 6 illustrates the phase ordering of flexible dimers, comparing three contrasting cases: a very stiff molecule $\left(\kappa=100 k_{B} T\right.$ with a correspondingly narrow distribution of bending angle fluctuations: standard deviation $\sigma_{\psi}=\sqrt{\left\langle\psi^{2}\right\rangle} \approx 8.5^{\circ}$ ); and a very flexible molecule $\left(\kappa=0.01 k_{B} T\right.$ and a practically flat distribution of bending angle fluctuations: standard deviation
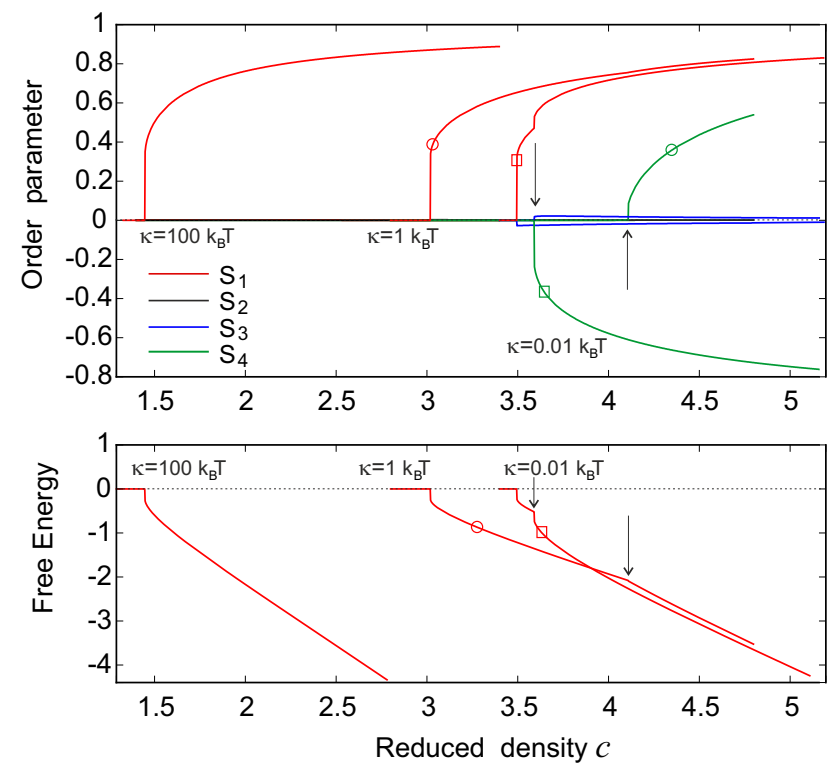

FIG. 6. Order parameters and excess free energy (in units of $\left.k_{B} T\right)$ plotted against reduced density for three dimers: stiff, with $\kappa=100 k_{B} T$ (plain curves); medium, with $\kappa=1 k_{B} T$ (curves labelled by $\odot$ ); and very flexible, with $\kappa=0.01 k_{B} T$ (curves labelled by $\square$ ). In all cases we find a strong firstorder transition to the prolate $\mathrm{N}_{\mathrm{U}}^{+}$phase, which in the case of non-stiff dimers is followed by a $\mathrm{N}_{\mathrm{U}}^{+}-\mathrm{N}_{\mathrm{B}}$ transition (marked by arrows).

$\left.\sigma_{\psi}=\sqrt{\left\langle\psi^{2}\right\rangle} \approx 52^{\circ}\right)$. A molecule with intermediate stiffness $\left(\kappa=1 k_{B} T\right.$ and $\left.\sigma_{\psi}=\sqrt{\left\langle\psi^{2}\right\rangle} \approx 43^{\circ}\right)$ is also included. For stiff dimers we find a single first-order transition to a prolate uniaxial $\mathrm{N}_{\mathrm{U}}^{+}$phase. Note that there is a nonzero equilibrium excess free energy arising from the $S_{5}$ term in the mean-field potential, which will always be present when incorporating the intra-molecular energy into Eqs. (20)-(21), and so an additive constant needs to be introduced to correctly compare $\Delta F$ in the three cases, Fig. $6(\mathrm{~b})$.

For $\kappa \leq k_{B} T$ we find a second transition, from the uniaxial nematic phase into a biaxial phase $\mathrm{N}_{\mathrm{B}}$, where the key order parameter $S_{4} \neq 0$. This is unexpected; unlike the case of the rigid bent rod of the previous section, the flexible dimer has no intrinsic biaxiality, with its equilibrium shape straight (at $\psi=0$ ). It nevertheless appears that, when the spread of dimer fluctuations becomes sufficiently wide, the system finds an advantage in restricting these fluctuations to a single plane instead of allowing the full range of the angle $\chi$ to be uniformly explored. We hypothesise that this is the origin of the biaxial order emerging at higher densities; ordering orientationally along one additional axis maximises free volume, as in the Onsager picture of the I-N transition. The two transitions are very close (i.e., the uniaxial nematic phase has a very narrow range) for flexible dimers, while for $\kappa=1 k_{B} T$, the two transitions rapidly diverge in reduced density, to between $c \approx 3.0$ and 4.1 (see Fig. 6 ).

The most remarkable feature of the results presented in 

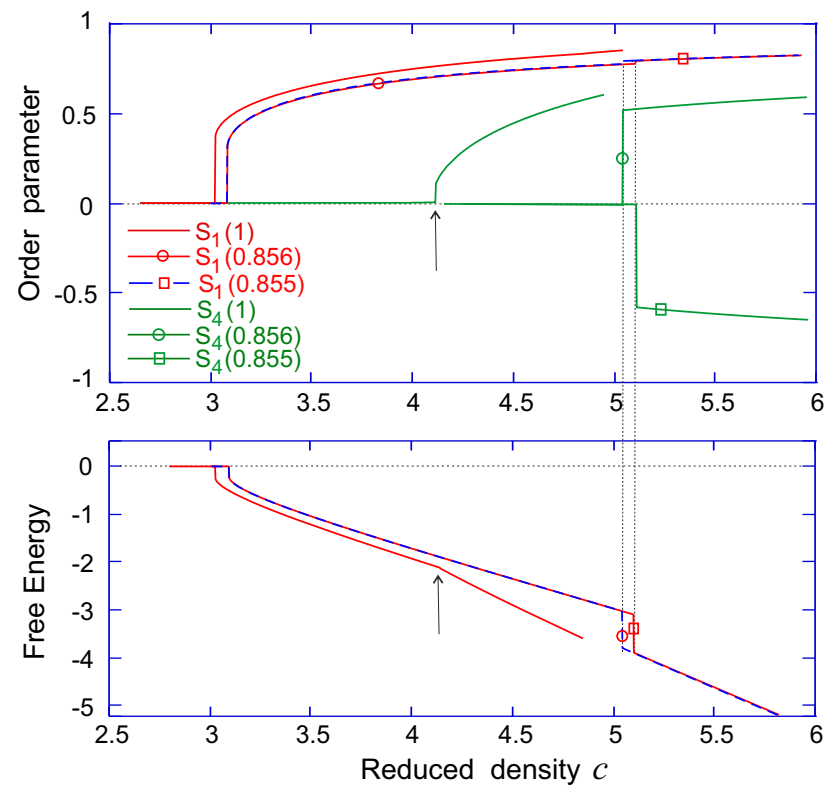

FIG. 7. Order parameters and excess free energy (in units of $k_{B} T$ ) plotted against reduced density for dimers with bending stiffness $\kappa$ crossing over from the $\mathrm{N}_{\mathrm{B}}^{+}$to the $\mathrm{N}_{\mathrm{B}}^{-}$phase. For $\kappa=0.855 k_{B} T$ (curves labelled by $\square$ ) the second transition is into the phase with a negative $S_{4}$; for $\kappa=0.856 k_{B} T$ (labelled by $\odot)$ the emerging phase has a positive $S_{4}$. The transitions at $\kappa=1 k_{B} T$ (plain curves) are also shown for reference; the biaxial transition for this bending stiffness is marked by the arrow).

Fig. 6 is the comparison of the transitions and phases at $\kappa=1 k_{B} T$ and $0.01 k_{B} T$. On increasing the density, both systems enter into a phase with positive order parameter $S_{1}$ : this is the uniaxial prolate nematic $\mathrm{N}_{\mathrm{U}}^{+}$. Then, the next transition takes the system into a biaxial nematic phase with a very small change in $S_{1}$, but with the rapidl y growing order parameter $S_{4}$. Surprisingly, there appear to be two different biaxial nematic phases - one with positive $S_{4}$, the other with negative $S_{4}$. It is tempting to relate these two phases to $\mathrm{N}_{\mathrm{B}}^{+}$and $\mathrm{N}_{\mathrm{B}}^{-}$, which we discussed in section III A (Fig. 5). There are, however, significant differences: here, the two other order parameters $\left(S_{2}\right.$ and $S_{3}$ ) remain zero, and the principal uniaxial order parameter $S_{1}$ remains positive. If we recall the definitions of our four order parameters, which are the averages of the Wigner functions, Eqs. (7), it should not surprise us that $S_{3}=0$, as this order parameter is a measure of molecular biaxiality, and a flexible dimer is not intrinsically biaxial because it can bend in any plane. On the other hand, $S_{2}$ is (like $S_{4}$ ) a measure of phase biaxiality; it describes the asymmetry of the fluctuations of the long molecular axis about the primary director. In the limit of strong uniaxial order $\left(S_{1} \rightarrow 1\right)$, which is approximately realised here, it can be proved [50] that $S_{2} \propto S_{4}\left(1-S_{1}\right)$ and $S_{2}$ is thus expected to be small.

Fig. 7 zooms into the crossover region where the switch between these $\mathrm{N}_{\mathrm{B}}^{+}$and $\mathrm{N}_{\mathrm{B}}^{-}$phases occurs, comparing sys- tems with $\kappa=0.855 k_{B} T$ and $0.856 k_{B} T$ (again, the case of $\kappa=1 k_{B} T$ is added for reference). We find a sharp discontinuous switch between negative and positive $S_{4}$, which then varies little on further density increase, associated with a clear jump in the free energy $\Delta F$ at the transitions, while having a negligible effect on the $S_{1,2,3}$ order parameters. Comparing these results with Fig. 6, we see that the 'strength' of this discontinuity at the $\mathrm{N}_{\mathrm{U}^{-}}$ $N_{\mathrm{B}}$ transition diminishes in both directions away from the critical value of $\kappa \approx 0.86 k_{B} T$. The fact that the $\mathrm{N}_{\mathrm{B}}^{+}$ and $\mathrm{N}_{\mathrm{B}}^{-}$phases have precisely the same free energy, and evidently $S_{4}$ in the $\mathrm{N}_{\mathrm{B}}^{+}$phase is simply $-S_{4}$ in the $\mathrm{N}_{\mathrm{B}}^{-}$ phase, suggests that $\mathrm{N}_{\mathrm{B}}^{+}$and $\mathrm{N}_{\mathrm{B}}^{-}$are not two physically distinct phases, but rather the same phase with the secondary director along the $x$ - and $y$-axes, respectively. In other words, when the $\mathrm{V}$-shaped molecule preferentially lies in the $x z$-plane, $S_{4}>0$, and when the V-shaped molecule is in the $y z$-plane, $S_{4}<0$, cf. the definition of $S_{4}$ in Eqs. (9).

The full phase diagram of the system in terms of the variables: (reduced) density $c$ vs. bending stiffness $\left(\kappa / k_{B} T\right)$ is shown in Fig. 8. This is a map of the parameter space where apparently four phases exist: isotropic, prolate uniaxial nematic, and the two biaxial nematic phases (which we assert is the same phase, with perpendicular principal axes). The topology of the $\mathrm{I}-\mathrm{N}_{U}^{+}$transition line is as expected: when the bending stiffness is very high, the dimer behaves as a rigid rod of length $2 L$; its ordering transition occurs at a lower reduced density $c \approx 1.32$, which matches exactly a separate calculation of the Onsager I-N transition of rigid rods for our choice of units (remember that our reduced density $c$, defined in Eq. (22), is constructed in such a way that results do not depend on the molecular volume $L^{2} D$, but the volume fraction $\varphi$ refers to individual rods of length $L$ ). In the opposite limit of a very flexible dimer, there is no corresponding straightforward a priori limit to be met. Even when the bending angle $\psi$ is not constrained by any energy penalty, the entropic effect of two connected rods delays the onset of orientational order, thereby dramatically driving up the transition density.

At a higher density and low bending stiffness, a second transition line, between the $\mathrm{N}_{\mathrm{U}}^{+}$and $\mathrm{N}_{\mathrm{B}}$ phases emerges. We labelled the two biaxial phases $\mathrm{N}_{\mathrm{B}}^{+}$and $\mathrm{N}_{\mathrm{B}}^{-}$in this map according to the sign of the $S_{4}$ order parameter. The $\mathrm{I}-\mathrm{N}_{\mathrm{U}}^{+}$and $\mathrm{N}_{\mathrm{U}}^{+}-\mathrm{N}_{\mathrm{B}}$ transitions appear to be very close as $\kappa \rightarrow 0$, and we are not certain whether this constitutes a single Landau point, which we miss for some reason of numerical fidelity, or whether the small separation of the two transitions is a real physical effect. In either case, we must remember that this physical system is very different from the naturally biaxial rigid bent-rod, and the nature of orientational ordering of two linked rods with no bending penalty is very different too.

We were able to numerically map the topology of the crossover region between $\mathrm{N}_{\mathrm{B}}^{+}$and $\mathrm{N}_{\mathrm{B}}^{-}$phases with some accuracy, as shown in the inset of Fig. 8. Again, we wish to be certain whether the two biaxial phases, $\mathrm{N}_{\mathrm{B}}^{+}$ 


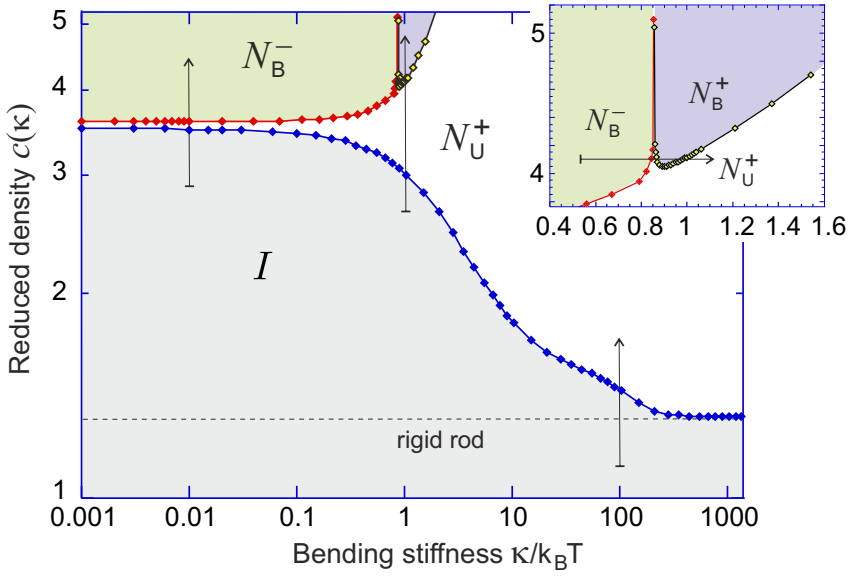

FIG. 8. Reduced density-bending stiffness phase diagram of flexible dimers. The $\mathrm{I}-\mathrm{N}_{\mathrm{U}}^{+}$phase transition boundary (lower curve) reaches the asymptote of $c \approx 1.35$ at very large $\kappa$. For small $\kappa$ the flexible dimers also form the biaxial phase at higher density. Regions corresponding to the $\mathrm{I}, \mathrm{N}_{\mathrm{U}}^{+}, \mathrm{N}_{\mathrm{B}}^{+}$and $\mathrm{N}_{\mathrm{B}}^{-}$phases are labeled in the figure. The vertical arrows mark the three density scans presented in Fig. 6. The horizontal arrow in the inset marks the stiffness scan explored in Fig. 9.

and $\mathrm{N}_{\mathrm{B}}^{-}$, are always separated by an infinitesimally thin region of uniaxial nematic phase, or if there is a direct transition between them. We therefore attempted to scan this phase map in a different direction: taking as constant the reduced density $c$ and varying the dimer stiffness $\kappa$ in great detail; the result is presented in Fig. 9. It is clear that there is a narrow region of uniaxial nematic phase separating the two versions of the biaxial phase (i.e. in order to transform from $\mathrm{N}_{\mathrm{B}}^{-}$to $\mathrm{N}_{\mathrm{B}}^{+}$the system needs to first melt into a higher-symmetry $\mathrm{N}_{U}$ phase). We carried out the same scan at a much higher density of $c=5$, finding the same (although narrower) region of the uniaxial nematic phase.

To understand this phase ordering, one needs to correlate the phases we observe with the theoretical analyses of Lubensky and Radzikhovsky [3], and of Luckhurst et al. [45], which establish the symmetries and possible transitions between many possible phases. Their analysis is based on phase symmetry, and therefore our biaxial phases (or just one fundamental phase $\mathrm{N}_{\mathrm{B}}^{ \pm}$) must be among their classification. However, linking the molecular shape with the phase symmetry, which is often the basis for understanding the phase ordering, is not straightforward since our molecules are not intrinsically biaxial, nor do they have a rigid shape. As such, neither theory $[3,45]$ applies directly to our system; the dynamical effect of the bending fluctuations of a flexible dimer on the equilibrium phase ordering is yet to be fully understood.

The bending stiffness $\kappa$ of the dimer is the second control parameter in this system (alongside the dimensionless reduced density $c$ ); we used its non-dimensional form $\kappa / k_{B} T$ to measure the strength of this interaction per molecule, and also to compare with the overall free
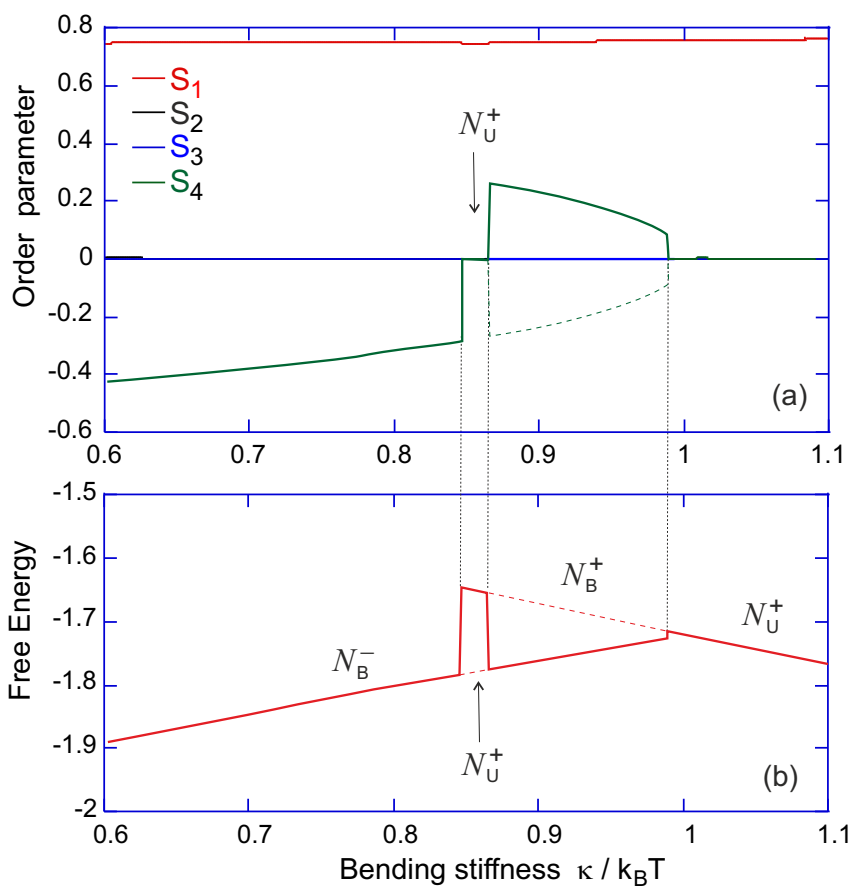

FIG. 9. 'Stiffness scan' of the phase diagram across the crossover region between the $\mathrm{N}_{\mathrm{B}}^{-}$to $\mathrm{N}_{\mathrm{B}}^{+}$phases, at $c=4.1$. Plot (a) shows the four order parameters, with the notable feature that the value of $S_{4}$ in $\mathrm{N}_{\mathrm{B}}^{+}$is just minus its continuation from $\mathrm{N}_{\mathrm{B}}^{-}$(as illustrated by the dashed line). Similarly, plot (b) shows the values of the free energy difference, where we see that the two biaxial phases, $\mathrm{N}_{\mathrm{B}}^{+}$and $\mathrm{N}_{\mathrm{B}}^{-}$have essentially the same free energy (by extrapolation). Likewise the free energy difference of the uniaxial phase $\mathrm{N}_{\mathrm{U}}$ sandwiched between the two biaxial phases is the continuation of that of the uniaxial phase at larger $\kappa$ (as illustrated by the dashed lines).

energy difference $\Delta F$ between the emerging phases presented in most plots. In a thermally-fluctuating system with unrestricted values of the variable $\psi$, the variance (standard deviation $\sigma_{\psi}$ ) of its equilibrium distribution would be given by $\sigma_{\psi}=\sqrt{k_{B} T / \kappa}$. Qualitatively, this would have to be the limiting case for very stiff dimers, for which the boundedness of the $\psi$-range is effectively never probed. However, for moderate and low values of $\kappa$, the finite range of $\psi\left(\psi \leq 90^{\circ}\right)$ will become significant; in the limiting case of $\kappa \rightarrow 0$, for which the fluctuations of $\psi$ are unrestricted, the variance saturates at $\sigma_{\psi}=\sqrt{\pi^{2} / 12}$. Our system is fully athermal, intended to be driven purely by the entropy of excluded volume, but the bending energy, Eq. (16), is added to the purely entropic effects. We have effectively included this potential energy alongside all other effects by scaling $\kappa / k_{B} T$. A very interesting feature needs to be highlighted: at our 'critical' value of stiffness $\kappa \approx 0.86 k_{B} T$ (where the crossover between $\mathrm{N}_{\mathrm{B}}^{+}$and $\mathrm{N}_{\mathrm{B}}^{-}$occurs) we calculate the distribution variance $\sigma_{\psi}$ to be almost exactly $45^{\circ}$. For $\kappa>0.86 k_{B} T$, i.e. in what we call the $\mathrm{N}_{\mathrm{B}}^{+}$phase with positive $S_{4}$, the fluctuating dimer retains an extended shape 
with a largely obtuse angle between the connected rods. Not surprisingly, we find the dimer principally aligned along $z$ with its azimuthal fluctuations biased in the $x y$ plane (the apex pointing along $x$ ). On the other hand, for $\kappa<0.86 k_{B} T$ (i.e. in the $\mathrm{N}_{\mathrm{B}}^{-}$region), the r.m.s. angle between the two rods is acute. We imagine that the $\mathrm{V}$ shaped (average) dimer aligns with its apex pointing up the $z$ axis, while the two rods preferentially reside in the $y z$ plane, producing the negative $S_{4}$.

Finally, a note of caution is in order - as detailed above, our theory does not reproduce the correct behavior when $\psi \rightarrow \pi / 2$. Very flexible dimers would have a noticeable probability weight corresponding to configurations in this range, and so the theory may become unreliable in this limit. This was mentioned above in the discussion of Fig. 8 when $\kappa \rightarrow 0$.

\section{CONCLUSIONS}

In this paper, the isotropic-nematic phase transitions of rigidly bent and flexible dimers were investigated using the Onsager second-virial theory of hard particle fluids in the manner of Straley, combined with a superposition approximation. The results of [8] were confirmed, and the continuous phase transition at the Landau point exhibited. Flexible dimers were studied by modifying Straley's method to include an intra-molecular energy term that penalises bending, the results obtained being justifiable on physical grounds. An apparently novel biaxial nematic phase of intrinsically uniaxial flexible dimers was found, although the molecules do not possess any permanent biaxiality. This new phase may, however, be an artefact of the approximations employed, for example the weak coupling between dimer stiffness and order parameters, and requires further investigation by either theory or simulation. The phase diagram of flexible dimers was plotted, which exhibits a non-trivial topology, with phase transitions between isotropic, prolate uniaxial nematic, and biaxial nematic phases, depending on the density and bond stiffness. A natural continuation of this work would be to investigate the effect of a non-zero equilibrium bond angle, $\psi_{0} \neq 0$, on the emergence and stability of various orientationally ordered phases of flexible dimers.

\section{ACKNOWLEDGMENTS}

P. I. C. Teixeira acknowledges financial support from the Fundação para a Ciência e Tecnologia of Portugal, under contracts nos. PEst-OE/FIS/UI0618/2014 and EXCL/FIS-NAN/0083/2012. A. Vaghela is grateful to St John's College and the Cavendish Laboratory for their support.
[1] T. Niori, T. Sekine, J. Watanabe, T. Furukawa, and H. Takezoe J. Mater. Chem. 6, 1231 (1996).

[2] H. Takezoe and Y. Takanishi, Jap. J. Appl. Phys. 45, 597 (2006).

[3] T. C. Lubensky and L. Radzihovsky, Phys. Rev. E 66, 031704 (2002).

[4] M. J. Freiser, Phys. Rev. Lett. 24, 1041 (1970).

[5] G. R. Luckhurst and T. J. Sluckin (eds.), Biaxial Nematic Liquid Crystals: Theory, Simulation and Experiment (Wiley, Chichester, 2015).

[6] M. Lehmann, Liq. Cryst. 38, 1389 (2001).

[7] J. P. Straley, Phys. Rev. A 10, 1881 (1974).

[8] P. I. C. Teixeira, A. J. Masters, and B. M. Mulder, Molec. Cryst. Liq. Crysf. 323, 167 (1998).

[9] M. A. Bates, Chem. Phys. Lett. 437, 189 (2007).

[10] L. A. Madsen, T. J. Dingemans, M. Nakata, and E. T. Samulski, Phys. Rev. Lett. 92, 145505 (2004).

[11] B. R. Acharya, A. Primak, and S. Kumar, Phys. Rev. Lett. 92, 145506 (2004).

[12] V. Prasad, S.-W. Kang, K. A. Suresh, L. Joshi, Q. Wang, and S. Kumar, J. Am. Chem. Soc. 127, 17224 (2005).

[13] C. D. Southern, P. D. Brimicombe, S. D. Siemianowski, S. Jaradat, N. Roberts, V. Görtz, J. W. Goodby and H. F. Gleeson, EPL 82, 56001 (2008).

[14] M. S. Park, B.-J. Yoon, J. O. Park, V. Prasad, S. Kumar, and M. Srinivasarao, Phys. Rev. Lett. 105, 027801 (2010).

[15] V. Görtz and J. W. Goodby, Chem. Commun. 26, 3262 (2005).
[16] B. Senyuk, H. Wonderly, M. Mathews, Q. Li, S. V. Shiyanovskii, and O. D. Lavrentovich, Phys. Rev. E 82, 041711 (2010).

[17] Y.-K. Kim, M. Majumdar, B. I. Senyuk, L. Tortora, J. Seltmann, M. Lehmann, A. Jákli, J. T. Gleeson, O. D. Lavrentovich, and S. Sprunt, Soft Matter 8, 8880 (2012).

[18] A. Ferrarini, G . R. Luckhurst, P. L. Nordio, and S. J. Roskilly, Chem. Phys. Lett., 214, 409 (1993).

[19] G. R. Luckhurst, Thin Solid Films 393, 40 (2001).

[20] G. R. Luckhurst, Angew. Chem. Int. Ed. 44, :2834 (2005).

[21] M. Lehmann, S.-W. Kang, C. Köhn, S. Haseloh, U. Kolb, D. Schollmeyer, Q.-B. Wang, and S. Kumar, J. Mater. Chem. 16, 4326 (2006).

[22] P. Grzybowski and L. Longa, Phys. Rev. Lett. 107, 027802 (2011).

[23] T. B. T. To, T. J. Sluckin, and G. R. Luckhurst, Liq. Cryst. 43, 1448 (2106).

[24] P. J. Camp, M. P. Allen, and A. J. Masters, J. Chem. Phys. 111, 9871 (1999).

[25] Y. Lansac, P. K. Maiti, N. A. Clark, and M. A. Glaser, Phys. Rev. E 67, 011703 (2003).

[26] A. Dewar and P. J. Camp, Phys. Rev. E 70, 011704 (2004).

[27] M. A. Bates and G. R. Luckhurst, Phys. Rev. E 72, 051702 (2005).

[28] E. M. Terentjev, C. Rosenblatt, and R. G. Petschek, J. Phys. II (France) 3, 41 (1993).

[29] E. M. Terentjev and R. G. Petschek, J. Phys. II (France) 3, 661 (1993). 
[30] A. C. Griffin, S. L. Sullivan, and W. E. Hughes, Liq. Cryst. 4, 667 (1989).

[31] D. J. Photinos, E. T. Samulski, and H. Toriumu, J. Chem. Phys. 94, 2758 (1991).

[32] N. J. Heaton and G. R. Luckhurst, Mol. Phys. 66, 65 (1989).

[33] G. R. Luckhurst, Liq. Cryst. 36, 1295 (2009).

[34] L. Onsager, Ann. N . Y. Acad. Sci. 51, 627 (1949).

[35] B. Mulder, Phys. Rev. A 39, 360 (1989).

[36] C. G. Gray and K. E. Gubbins, Theory of Molecular Flu$i d s$, Vol. 1 (Clarendon Press, Oxford, 1984).

[37] M. P. Allen, D. Frenkel, G. T. Evans and B. M. Mulder, Adv. Phys. Chem. 86, 1452 (1993).

[38] P. Bolhuis and D. Frenkel, J. Chem. Phys. 106, 686 (1997).

[39] F. Bisi and R. Rosso, Eur. J. Appl. Math. 23, 29 (2012).

[40] G. J. Vroege and H. N. W. Lekkerkerker, Rep. Prog. Phys. 55, 1241 (1992).
[41] B. Tjipto-Margo and G. T. Evans, J. Chem. Phys. 93, 4254 (1990).

[42] F. Bisi, R. Rosso, E. G. Virga, and G. E. Durand, Phys. Rev. E 78, 011705 (2008).

[43] R. Rosso, Liq. Cryst. 34, 737 (2007).

[44] B. Méttout, Phys. Rev. E 72, 031706 (2005).

[45] G. R. Luckhurst,S. Naemura,T. J. Sluckin, T. B. T. To, and S. Turzi, Phys. Rev. E 84, 011704 (2011).

[46] J.-L. Colot, X.-G. Wu, H. Xu, and M. Baus, Phys. Rev. A 38, 2022 (1988).

[47] W. H. Press, S. A. Teukolsky, W. T. Vetterling , and B. P. Flannery, Numerical Recipes: The Art of Scientific Computing 2nd edn. (Cambridge University Press, Cambridge, 1992).

[48] B. Tjipto-Margo and G. T. Evans, J. Chem. Phys. 94, 4546 (1991).

[49] A. I. Larkin and S. A. Pikin, Sov. Phys. JETP 29, 891 (1969).

[50] M. A. Osipov, T. J. Sluckin, and E. M. Terentjev, Liq. Cryst. 19, 197 (1995). 